\title{
Broadband Dispersion Characterization of Chalcogenide Tapered Photonic Crystal Fiber
}

\author{
Svyatoslav Kharitonov*, Sida Xing and Camille-Sophie Brès \\ Ecole Polytechnique Fédérale de Lausanne,Photonic Systems Laboratory, PHOSL-STI-IEL, Station 11, CH-1015 Lausanne, Switzerland \\ *svyatoslav.kharitonov@epfl.ch
}

\begin{abstract}
Group-velocity dispersion of birefringent GeAsSe tapered PCF is directly measured over 1900-2300nm range using all-fiber Mach-Zehnder interferometer. We experimentally prove that zero-dispersion wavelength of chalcogenide PCFs can be shifted to thulium/holmium doped silica emission band.

OCIS codes: (060.2270) Fiber characterization; (260.2030) Dispersion; (060.2390) Fiber optics, infrared
\end{abstract}

\section{Introduction}

Chalcogenide (ChG) glass fibers represent an attractive waveguide platform for optical parametric processes in midinfrared (MIR) range due to their strong nonlinearity and wide transparency window, spanning up to $15 \mu \mathrm{m}$, depending on the glass composition [1]. Fiber optical parametric oscillators (OPO)[2] and wavelength converters in MIR [3] based on ChG microwires, were reported. Moreover, supercontinuum generation (SCG) in ChG fibers within 1.4-13.3 $\mu \mathrm{m}$ [4] and 2.0-15.1 $\mu \mathrm{m}$ bands [1], pumped using solid-state OPOs at 4.3-6.5 $\mu \mathrm{m}$ and $9.8 \mu \mathrm{m}$, respectively, have been recently demonstrated. In most applications, by manipulating the fiber index profile the dispersion could be tailored around the pump wavelength to ensure an efficient excitation of parametric processes. GeAsSe hexagonal photonic crystal fiber (PCF) with a core diameter of $1.5 \mu \mathrm{m}$ and air hole diameter-to-pitch ratio of 0.58 was predicted to have two zero-dispersion wavelengths (ZDW), one at $2.15 \mu \mathrm{m}$ overlapping with the emission band of thulium-holmium doped fiber lasers, and one at $2.54 \mu \mathrm{m}$ [5]. Such structure can be obtained via a two-step manufacturing method (drawing and tapering) that, however, can lead to imperfections in the PCF lattice and introduces birefringence. Physically, it implies some slight radial variations of the cladding air hole sizes, leading to a different effective diameter-to-pitch ratio depending on the input light polarization. Retrieving the dispersion properties of designed ChG fibers, either by linear [6] or nonlinear methods [7], is therefore important.

The linear low-coherence interferometry (LCIM) represents the most suitable tool for broadband characterization of short fibers (up to few tens of meters) [6]. It was applied to measure the group velocity dispersion (GVD) of stepindex ChG fiber in 3.0-4.6 $\mu \mathrm{m}$ spectral region using free-space Mach-Zehnder interferometer (MZI) [4]. A ChG PCF was characterized in 1.2-1.7 $\mu \mathrm{m}$ band using silica all-fiber MZI [8] that is significantly more robust and easier to operate compared to its free-space counterpart. However, the implementation of LCIM with monolithic MZI for wavelengths longer than $2.1 \mu \mathrm{m}$ is impeded by the exponentially increasing attenuation of fused silica, which limits both the bandwidth of supercontinuum (SC) sources, used as low-coherence input signal, and the transmission through the MZI arms. To partially overcome this limitation, thulium-doped fibers (TDF) can be used as a nonlinear medium, where light emission and amplification by the ${ }^{3} \mathrm{~F}_{4}-{ }^{3} \mathrm{H}_{6}$ and ${ }^{3} \mathrm{H}_{4}-{ }^{3} \mathrm{H}_{5}$ transitions, centered at 1.9 and $2.3 \mu \mathrm{m}$ wavelength, respectively, assists the SC generation up to $2.6 \mu \mathrm{m}$ [9].

In this paper, we present results on the dispersion characterization of GeAsSe tapered PCF fiber, which was designed to possess a ZDW around $2.2 \mu \mathrm{m}$. We evaluated the ZDW of $2230 \mathrm{~nm}$ and $2265 \mathrm{~nm}$ along two distinguished polarization axes. To the best of our knowledge, it is the first direct measurement that confirms shifting of ZDW of ChG PCF below $2.3 \mu \mathrm{m}$, approaching the emission band of thulium- and holmium-doped silica fiber lasers. The tests were performed over a continuous 1.9-2.3 $\mu \mathrm{m}$ band using monolithic MZI setup, resulting in a robust and simplified characterization. A custom broadband input signal source, relying on cascaded SCG in dispersion-shifted fiber (DSF) and TDF, was assembled from commercial off-the-shelf fiber optical components, and provided high power and $10 \mathrm{~dB}$ spectrum flatness over about $500 \mathrm{~nm}$.

\section{Low-coherence interferometry principles and experimental setup}

In LCIM, a broadband optical signal is passed through the MZI. One arm includes the fiber under test (FUT). The other arm acts as a reference (REF) composed of a fiber with known characteristics and a tunable optical delay line (ODL). The combined signals are sent to the optical spectrum analyzer (OSA) where the resulted interference fringes pattern $I \sim \cos \varphi(\omega)$ is recorded, with $\varphi(\omega)$ as arms phase difference, tailored around a certain angular frequency $\omega_{0}$ :

$$
\varphi(\omega)=\varphi_{0}+\left(\beta_{1}^{F U T} L^{\mathrm{FUT}}-\beta_{1}^{R E F} L^{R E F}-\tau\right) \Delta \omega+\left(\beta_{2}^{F U T} L^{F U T}-\beta_{2}^{R E F} L^{R E F}\right) \Delta \omega^{2} / 2+\left(\beta_{3}^{F U T} L^{F U T}-\beta_{3}^{R E F} L^{R E F}\right) \Delta \omega^{3} / 6+\ldots
$$


In Eq.(1), $\Delta \omega=\omega-\omega_{0}, \varphi_{0}$ a constant phase shift, $\beta_{n}$ the $n^{\text {th }}$-order dispersion, $\tau$ a delay introduced by the ODL. The condition $d \varphi / d \omega=0$ corresponds to the position of the broadest, central fringe of the interference pattern [6]:

$$
d \varphi / d \omega=0 \Rightarrow \omega=\omega_{0} \quad \text { and } \quad \beta_{1}^{\text {FUT }} L^{\text {FUT }}=\beta_{1}^{R E F} L^{R E F}+\tau .
$$

Eq. (2) implies that $\varphi(\omega)$ tailored around the central fringe frequency $\omega_{0}$ does not contain a linear term. For the dispersion evaluation, the phase of interferogram $\varphi(\omega)$ can be retrieved, fitted with a polynomial, and $\beta_{2}^{\text {FUT }}$ can be calculated from corresponding polynomial quadratic coefficient. Moreover, if the FUT exhibits some non-negligible birefringence, at the fixed delay $\tau$ the frequency of central fringe $\omega_{0}$ depends on the polarization of the input signal.
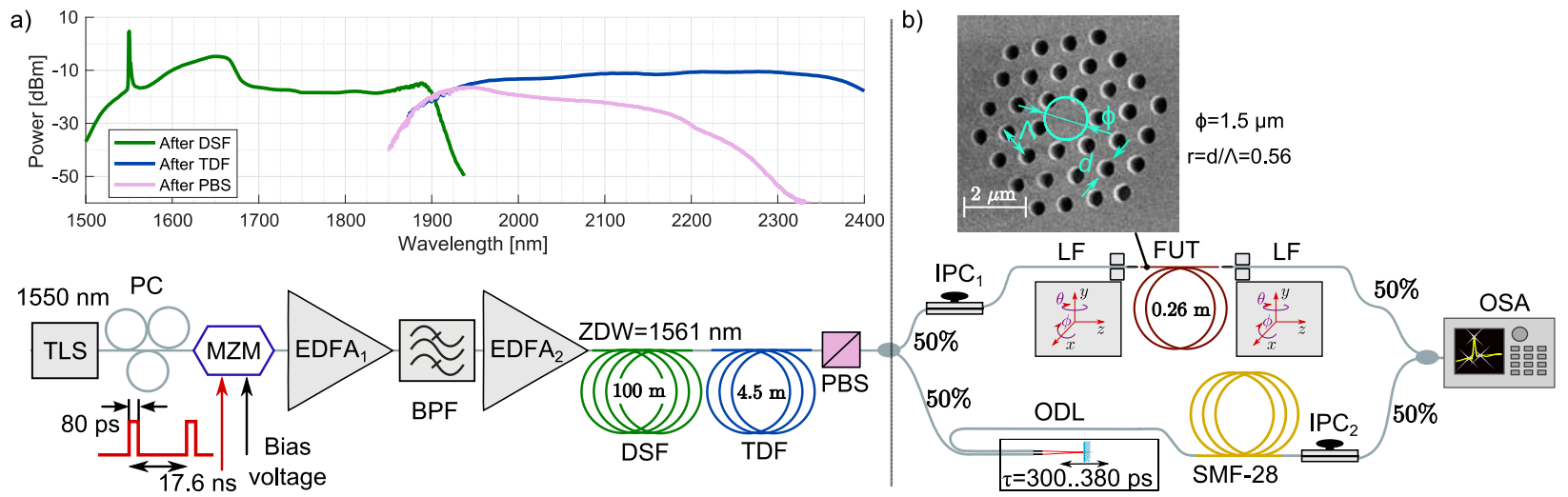

Fig. 1: Experimental setup: a) SC source, b) MZI layout. TLS: tunable laser source; PC: polarization controller; MZM: Mach-Zehnder modulator; BPF: bandpass filter; EDFA: erbium-doped fiber amplifier; DSF: dispersion-shifted fiber; TDF: thulium-doped fiber; PBS: polarization beam splitter; LF: lensed fiber; IPC: inline PC. Inset in (a): generated spectra, recorded at the different points of SC source. Inset in (b): SEM image of the PCF cross-section, indicating main geometry parameters - core diameter $\phi$, air hole diameter $d$ and pitch $\Lambda$.

The experimental setup consists of two main parts: a custom SC source (Fig. 1a), and an all-fiber MZI (Fig. 1b). For SCG, a pulsed pump signal (sub-100 W peak power) is coupled to $100 \mathrm{~m}$ long dispersion-shifted fiber (DSF), with ZDW at $1561 \mathrm{~nm}$. The pump wavelength is set at $1550 \mathrm{~nm}$ in order to generate an initial SC signal, spanning up to $1900 \mathrm{~nm}$, which is consequently sent into a $4.5 \mathrm{~m}$ long section of commercially available TDF (TmDF200 by OFS Fitel, Denmark). The SC part within 1.5-1.7 $\mu \mathrm{m}$ band is used to excite thulium cations. Pulses around $1.9 \mu \mathrm{m}$ trigger SCG in the TDF, supported by the light emission and amplification in ${ }^{3} \mathrm{~F}_{4}-{ }^{3} \mathrm{H}_{6}$ and ${ }^{3} \mathrm{H}_{4}{ }^{3} \mathrm{H}_{5}$ transitions, centered at 1.9 and $2.3 \mu \mathrm{m}$ [9]. As a result, a stable 10-dB flat SC spanning the 1.9-2.4 $\mu \mathrm{m}$ range is generated (see Fig 1 inset). However, the effective bandwidth of the dispersion measurements is limited to the 1.9-2.3 $\mu \mathrm{m}$ due to the bandwidth of the fiberized polarization beam splitter (PBS) used at the output of the source.
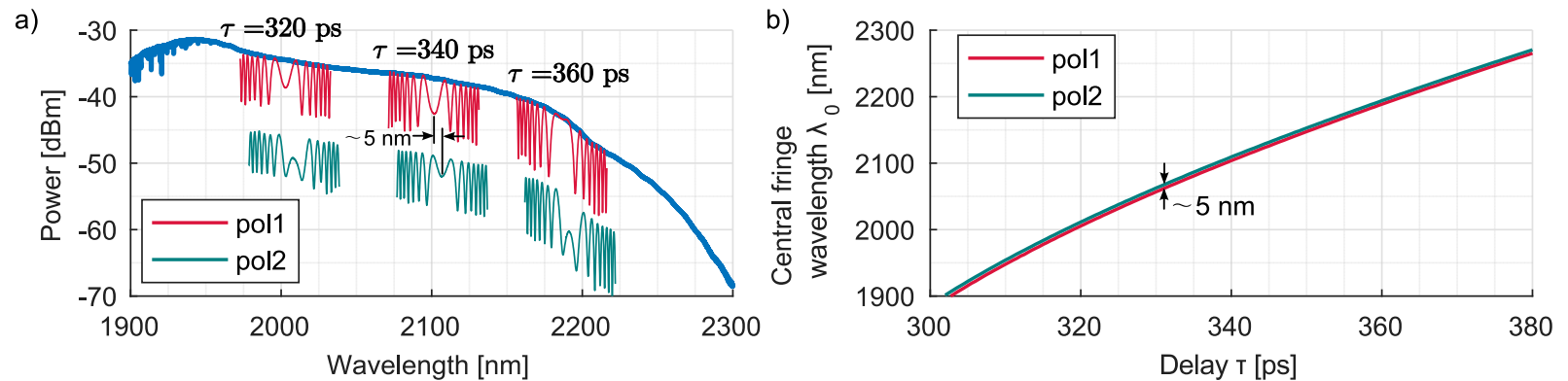

Fig. 2: a) TDF SC spectrum, superimposed with interferograms for chosen values of $\tau$ at two different input signal polarizations (pol1 and pol2). Interferograms for pol2 are artificially off-set for better visibility; b) central fringe position $\lambda_{0}(\tau)$.

The SC signal is sent to the MZI which includes the $0.26 \mathrm{~m}$ long section of GeAsSe tapered PCF (core diameter $\phi$ of $1.5 \mu \mathrm{m}$, and air holes diameter-to-pitch ratio $r=d / \Lambda$ of 0.56 ). The junctions between the lensed fibers (3.5 $\mu \mathrm{m}$ spot size in focus) and the FUT are the only free-space optics interfaces in the setup. Coupling losses are estimated of about $4 \mathrm{~dB}$ per facet, and propagation losses in the FUT are measured as $0.6 \mathrm{~dB} / \mathrm{m}$ at $1550 \mathrm{~nm}$. The reference arm contains a pigtailed motorized tunable optical delay line (ODL), and an additional section of SMF-28 for average balancing of optical paths in MZI. Low-insertion loss (less than $0.5 \mathrm{~dB}$ ) inline polarization controllers (IPC) are included in both MZI arms. The IPC 1 in the test arm is used prior to the FUT to align the input polarization state until it coincides with one of the birefringence axes of PCF and clear interference pattern is observed, as shown in Fig. 2a. The IPC $\mathrm{C}_{2}$ in the REF arm co-polarizes the interacting signals at the MZI output, maximizing the fringes 
contrast. The ODL time delay $\tau$ was swept to shift the central fringe wavelength $\lambda_{0}$ over the bandwidth of the SC (Fig. 2b). For each $\tau$ value, two different $\lambda_{0}$ were evaluated depending on the input polarization, indicating two distinct polarization axes.

\section{Dispersion characterization results and conclusions}

The dispersion measurement results are shown in Fig. 3. The GVD coefficients $\beta_{2}$ at two different polarization states of the input SC were directly retrieved from the interferograms, without applying any data smoothening algorithms. A strongly normal behavior at the blue edge of the spectral range is observed, gradually decreasing and reaching ZDW at $2230 \mathrm{~nm}$ and $2265 \mathrm{~nm}$, depending on the polarization state. The experimental data are overall in a good agreement with theoretical dispersion relations, calculated in COMSOL for two isotropic GeAsSe PCFs with $\mathrm{r}=0.562$ and $\phi=1.6 \mu \mathrm{m}$ and $\mathrm{r}=0.56$ and $\phi=1.5 \mu \mathrm{m}$. As it can be seen from the simulated $\beta_{2}$ curves, truly small fluctuations of the core diameter $\phi$, or air holes diameter-to-pitch ratio $r=d / \Lambda$ can drastically change the PCF dispersion properties, shifting the ZDW toward longer wavelength, or turning the PCF to all-normal dispersion fiber. For real fibers, where geometry parameters may vary radially, one PCF can combine diverse dispersion properties on different polarization axes. Discrepancy between experiment and simulation can be attributed to Sellmeier coefficients, which is taken from another GeAsSe glass with slightly different component ratio.

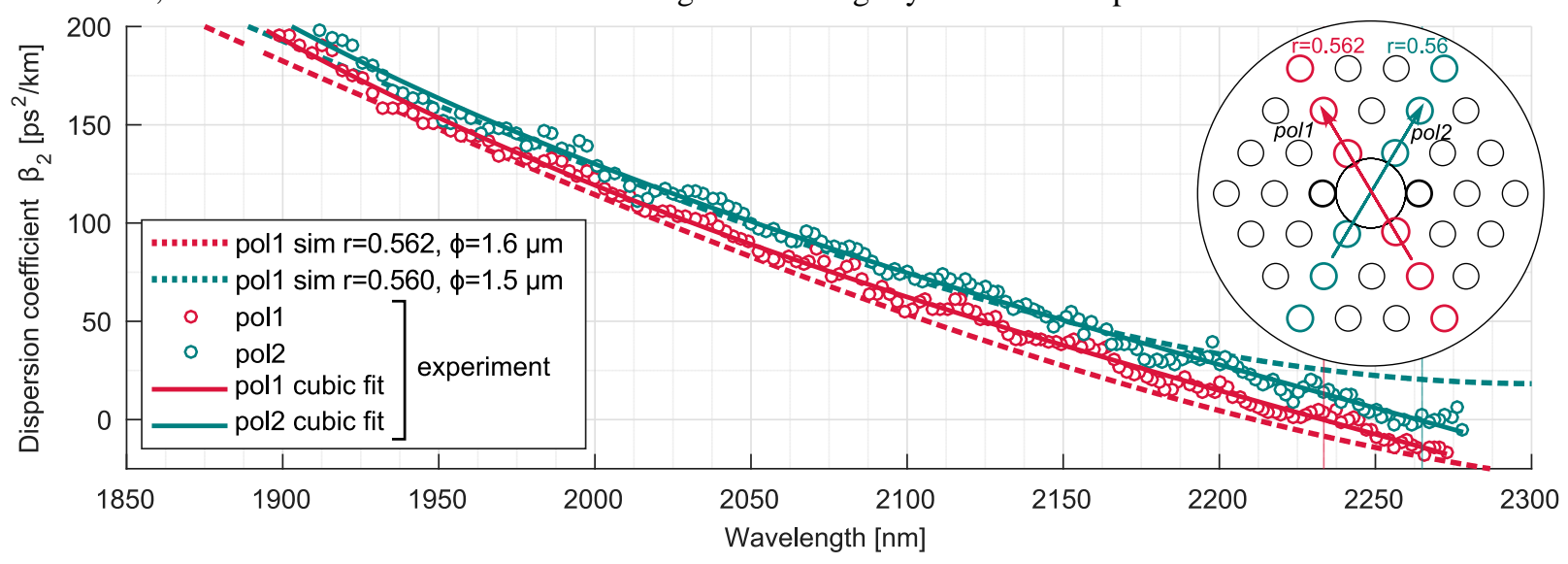

Fig. 3: Dispersion characterization results: GVD coefficient $\beta_{2}$, measured at two different polarization states (markers). Inset: schematic crosssection of the PCF, indicating two possible polarization axes, aligned with arrays of air holes of slightly different diameters. Dashed lines: dispersion relations, calculated in COMSOL for two isotropic ChG PCFs with $\mathrm{r}=0.562$ and $\phi=1.6 \mu \mathrm{m}$ and $\mathrm{r}=0.56$ and $\phi=1.5 \mu \mathrm{m}$.

In conclusion, we experimentally measured the dispersion of a birefringent GeAsSe tapered PCF fiber, using monolithic MZI setup. The fiber exhibits a ZDW at $2230 \mathrm{~nm}$ or $2265 \mathrm{~nm}$ along two distinguished polarization axes. Based on our simulations, we expect second ZDW located around $2.5 \mu \mathrm{m}$ for each polarization. To the best of our knowledge, it is the first direct experimental proof that the ZDW of ChG PCF can be shifted below $2.3 \mu \mathrm{m}$, approaching the emission band of thulium- and holmium-doped silica fiber lasers.

This work is supported in part by the European Research Council under grant agreement ERC-2012-StG306630-MATISSE

\section{References}

[1] T. Cheng et al, “Mid-infrared supercontinuum generation spanning 2.0 to $15.1 \mu \mathrm{m}$ in a chalcogenide step-index fiber,” Opt. Lett. 41, 2117 (2016).

[2] N. Abdukerim et al, “Chalcogenide-based optical parametric oscillator at $2 \mu \mathrm{m}$,” Opt. Lett. 41, 4364 (2016).

[3] L. Li et al, “Mid-infrared wavelength conversion from As2 Se3 microwires,” Opt. Lett. 42, 639 (2017).

[4] C. R. Petersen et al, "Mid-infrared supercontinuum covering the 1.4-13.3 $\mu$ m molecular fingerprint region using ultra-high NA chalcogenide step-index fibre,” Nat. Photonics 8, 830-834 (2014).

[5] S. Xing et al "Characterization and modeling of microstructured chalcogenide fibers for efficient mid-infrared wavelength conversion,” Opt. Express 24, 9741 (2016).

[6] H.-T. Shang, "Chromatic dispersion measurement by white-light interferometry on metre-length single-mode optical fibres,” Electron. Lett. 17, 603 (1981)

[7] B. Auguie et al, "Ultralow chromatic dispersion measurement of optical fibers with a tunable fiber laser," IEEE Photonics Technol. Lett. 18, 1825-1827 (2006).

[8] J. Fatome et al, “Linear and Nonlinear Characterizations of Chalcogenide Photonic Crystal Fibers,” J. Light. Technol. 27, 1707 (2009).

[9] J. Geng et al, "High-spectral-flatness mid-infrared supercontinuum generated from a Tm-doped fiber amplifier.,” Appl. Opt. 51, 834 (2012). 